\title{
Biomarkers and the Future of Pediatric Gastroenterology
}

\author{
Lindsay M Moye ${ }^{1}$, Jon Marc Rhoads² and Yuying Liu ${ }^{2}$ \\ ${ }^{1}$ Department of Pediatric Gastroenterology, University of Texas Health Science Center, USA \\ ${ }^{2}$ Division of Pediatric Gastroenterology, University of Texas Health Science Center, USA
}

Received: October 11, 2017; Published: October 13, 2017

*Corresponding author: Lindsay M. Moye, Department of Pediatric Gastroenterology, University of Texas Health Science Center at Houston, 6431 Fannin, MSB 3.137, Houston, TX, 77030, USA;

\begin{abstract}
Biomarker discovery in the field of pediatric gastroenterology is necessary so that clinicians can use objective, non-invasive ways to screen for a disease in its preclinical stage, measure disease severity or monitor response to a particular treatment. The utilization of high-throughput analyses, such as with proteomics and metabolomics, allows researchers to quickly investigate numerous methodologies and generate large datasets, while only requiring small quantities of the biological specimen.
\end{abstract}

Keywords: Biomarker; Proteomics; Metabolomics; High Throughput

Abbreviations: CD: Crohn's Disease; HC: Healthy Controls

\section{Introduction}

The term "biomarker" is used to describe any substance, structure or process that can be measured in the body or its products, and influence or predict the incidence of outcome or disease [1]. Be it carbohydrate, protein, lipid or gene (to name a few), biomarkers provide insight into disease detection, progression, and response to therapy [2]. The ideal biomarker is simple to collect, non-

invasive for the patient, while also being specific, objective, precise, reliable and inexpensive to analyze. An example of a biomarker with excellent sensitivity and specificity is fecal calprotectin for children with inflammatory bowel disease. The neutrophil product, a secreted antimicrobial product, is increasingly being used as a non-invasive marker for active disease and relapse [3,4].

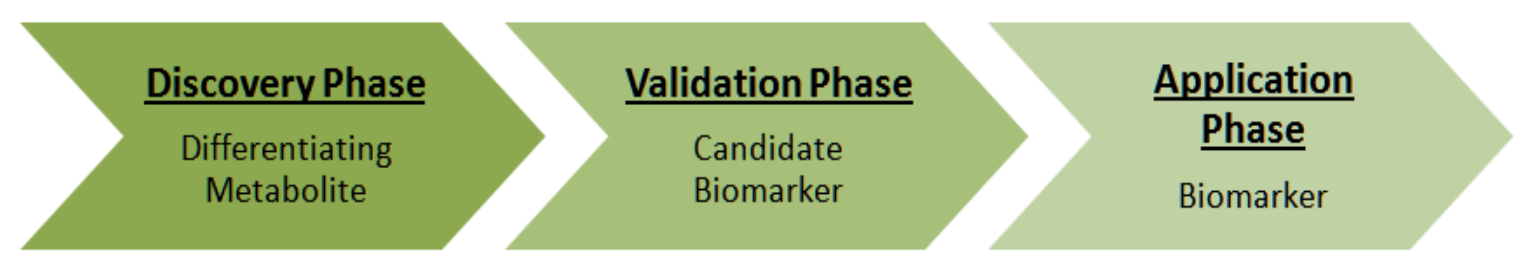

Figure 1: The process of biomarker discovery using high-throughput technologies. Adapted from Koulman et al [5].

Emerging advances in technologies and methodologies have contributed to the increase in biomarker discovery research. Contrary to more classical research design where there is a specific molecule or pathway in mind, untargeted analyses are becoming more commonplace [5]. High-throughput techniques allow investigators to cast a wide net around potential biomarkers in the hopes of finding significant differences between the specific groups, followed by testing those candidate biomarkers on larger cohorts. Having this multistep qualification process is also more desirable because it circumvents the challenges associated with expensive tests and obtaining an adequate population size (Figure 1).

\section{Pediatric Gastroenterology}

Biomarker research is particularly important in pediatric gastroenterology. Bio-fluids like blood, saliva, urine and stool are generally more practical to obtain than tissue biopsies. Gastrointestinal endoscopy with biopsy in children usually requires anesthesia, increased risks and social challenges such as parents taking time off from work. While questionnaires are cost-effective, quick and usually readily available, their application is limited in pediatrics when patients are nonverbal or not developmentally able to communicate. Questionnaires and Likert scales also suffer from recall bias and results can be difficult to interpret. An attractive 
method for monitoring disease progression or treatment response is one that can be conveniently done in clinic or the patient's home not require skill to collect and produces a specimen that remains biologically stable until submission for analysis.

Several notable articles have been published within the past decade with this end goal in mind.

i. Using urinary amino acid metabolomic profiling, Yan identified the urinary glutamine to glutamic acid ratio to be a promising biomarker in the discovery phase for distinguishing suspected pediatric chronic intestinal pseudo-obstruction from simple short bowel syndrome [6].

ii. In pediatric inflammatory bowel disease, detailed studies have shown an altered fecal microbial community, compared to healthy controls. However, with significant overlap with children without active disease [7]. These results lend credence, without absolute proof, to the concept of microbial participation in immune activation and contribution to pathogenesis of the disease. However, Ahmed et al recently examined potential differences between stools of patients with active Crohn's disease (CD) and healthy controls (HC), using fecal metabolite analysis by micro-extraction, gas chromatography/mass spectrometry and analysis by partial least squares discriminate analysis [8]. Their results indicated that there was almost perfect separation of fecal metabolites between CD patients and HC, but there were also significant differences between CD patients with inactive and active disease. The most impacted metabolites in active CD were heptanal and 1-octen-3-ol (increased) and methanethiol (decreased). These results suggest that metabolomic profiling may help, not only in studying disease pathogenesis, but also in identifying remission versus exacerbation.

iii. Other examples of pediatric gastrointestinal diseases that may be better understood using metabolites as disease markers are infantile colic, with elevated urinary 5-hydroxy indole acetic acid (a serotonin metabolite) [9] and celiac disease, which is characterized metabolically by reduced serum citrulline, choline, creatinine, branch chain amino acids and lipids [10]. These markers appear to be abnormal even before onset of the disease.

iv. One study currently in the discovery phase is comparing plasma and urine metabolomics in children with eosinophilic esophagitisto children without the disease. This cohort of patients would benefit greatly from a reliable biomarker of disease activity as absence of symptoms does not always correlate with disease remission, and chronic esophageal eosinophilia may lead to an increased risk for esophageal tears, perforation, food impactions and strictures [11].

\section{Conclusion}

As scientific, statistical and computer technologies continue to advance, so will the future of biomarker research. Previous "understand then investigate" methods may be replaced by an "investigate then understand" approach. Not only will the pathogenesis of pediatric gastrointestinal diseases be better understood, but significant progress will also be made in drug development and patient outcomes.

\section{References}

1. WTO (2001) WTO International Programme on Chemical Safety Biomarkers in Risk Assessment: Validity and Validation. WHO, Geneva, Switzerland.

2. Mayeux R (2004) Biomarkers: potential uses and limitations. NeuroRx 1(2): 182-188.

3. Henderson P, Casey A, Lawrence SJ, Kennedy NA, Kingstone K, et al. (2012) The diagnostic accuracy of fecal calprotectin during the investigation of suspected pediatric inflammatory bowel disease. Am J Gastroenterol 107(6): 941-949.

4. Pang T, Leach ST, Katz T, Day AS, Ooi CY (2014) Fecal biomarkers of intestinal health and disease in children. Front Pediatr 2: 6.

5. Koulman A, Lane GA, Harrison SJ, Volmer DA (2009) From differentiating metabolites to biomarkers. Anal Bioanal Chem 394(3): 663-670.

6. Yan JK, Zhou KJ, Huang JH, Wu QQ Zhang T, et al. (2017) Urinary glutamine/glutamate ratio as a potential biomarker of pediatric chronic intestinal pseudo-obstruction. Orphanet J Rare Dis 12(1): 62.

7. Gevers D, Kugathasan S, Denson LA, Vazquez Baeza Y, Van TW, et al. (2014) The treatment-naive microbiome in new-onset Crohn's disease. Cell Host Microbe 15(3): 382-392.

8. Ahmed I, Greenwood R, Costello B, Ratcliffe N, Probert CS (2016) Investigation of faecal volatile organic metabolites as novel diagnostic biomarkers in inflammatory bowel disease. Aliment Pharmacol Ther 43(5): 596-611.

9. Kurtoglu S, Uzum K, Hallac IK, Coskum A (1997) 5-Hydroxy-3-indole acetic acid levels in infantile colic: is serotoninergic tonus responsible for this problem? Acta Paediatr 86(7): 764-765.

10. Bernini P, Bertini I, Calabro A, la MG, Lami G, etal. (2011) Are patients with potential celiac disease really potential? The answer of metabonomics. J Proteome Res 10: 714-721.

11. Furuta GT, Liacouras CA, Collins MH, Gupta SK, Justinich C, et al. (2007) Eosinophilic esophagitis in children and adults: a systematic review and consensus recommendations for diagnosis and treatment. Gastroenterology 133(4): 1342-1363.

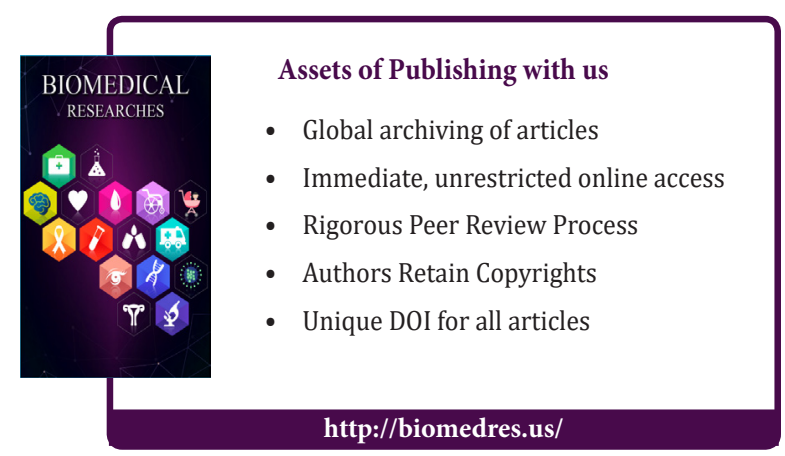

